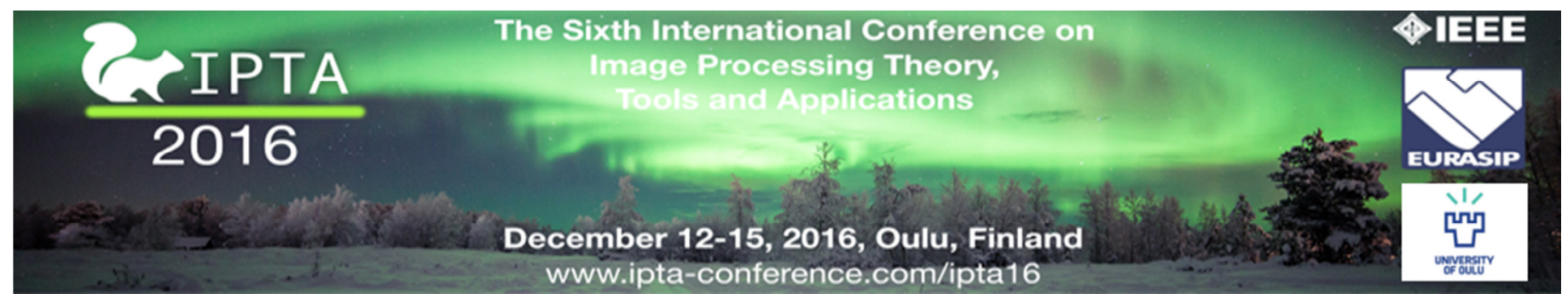

\title{
Conference Program
}

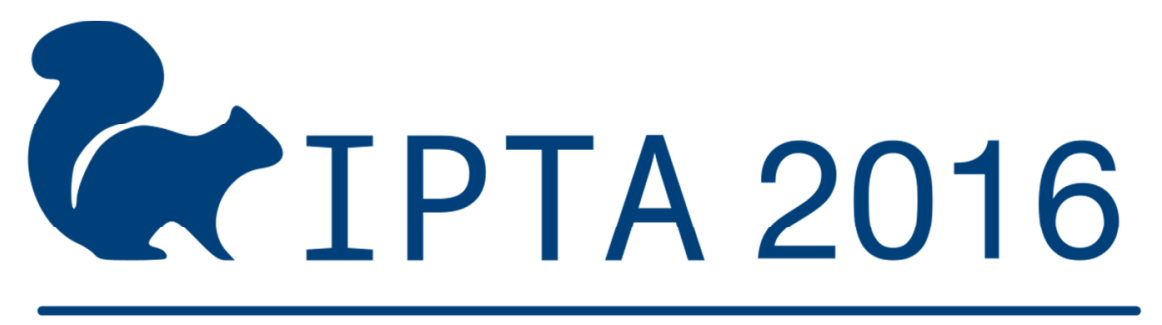

The sixth International Conference on Image Processing Theory, Tools and Applications December 12-15, 2016 Oulu, Finland

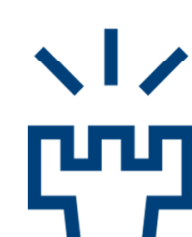

UNIVERSITY OF OULU IIEEE
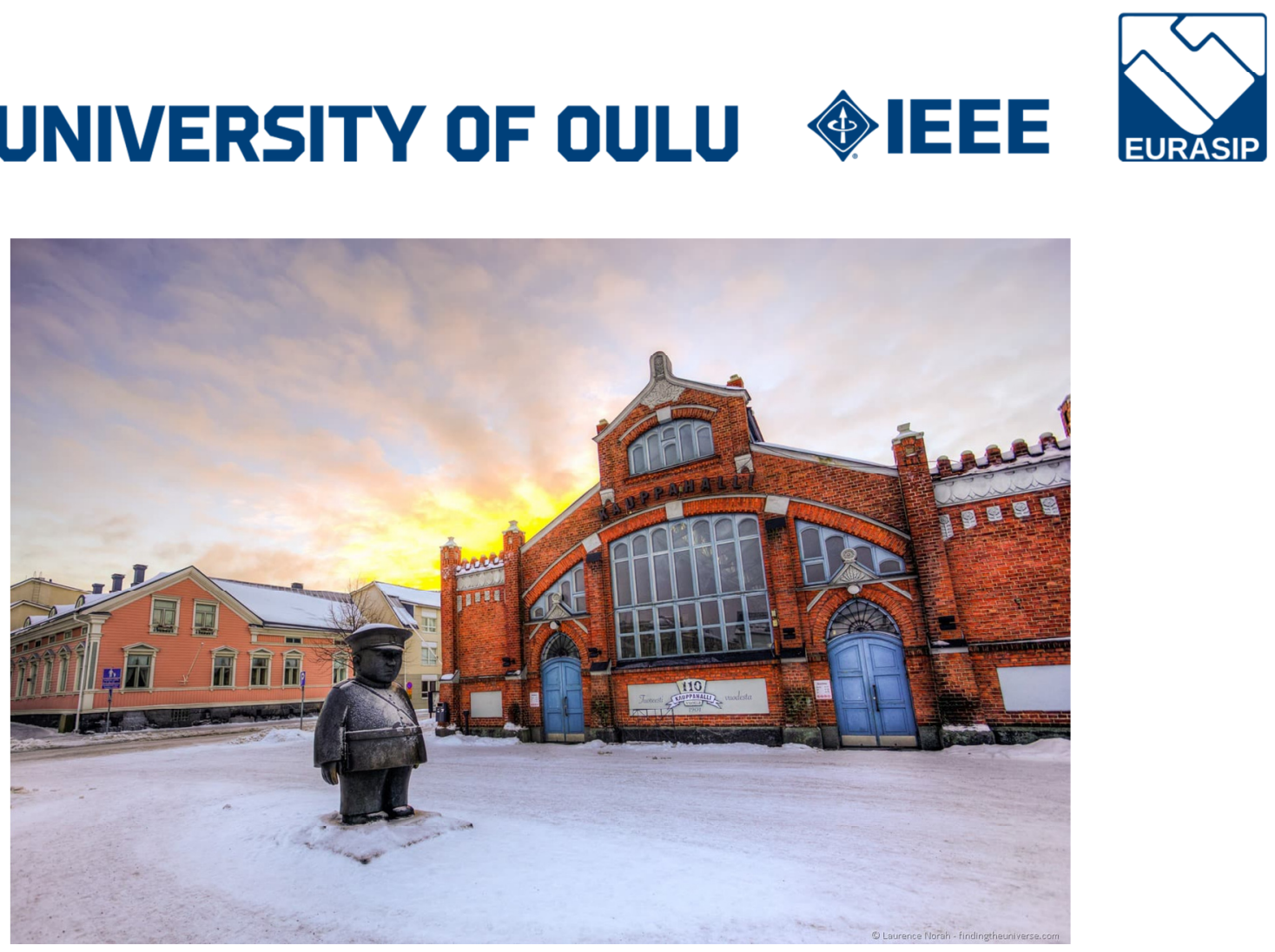


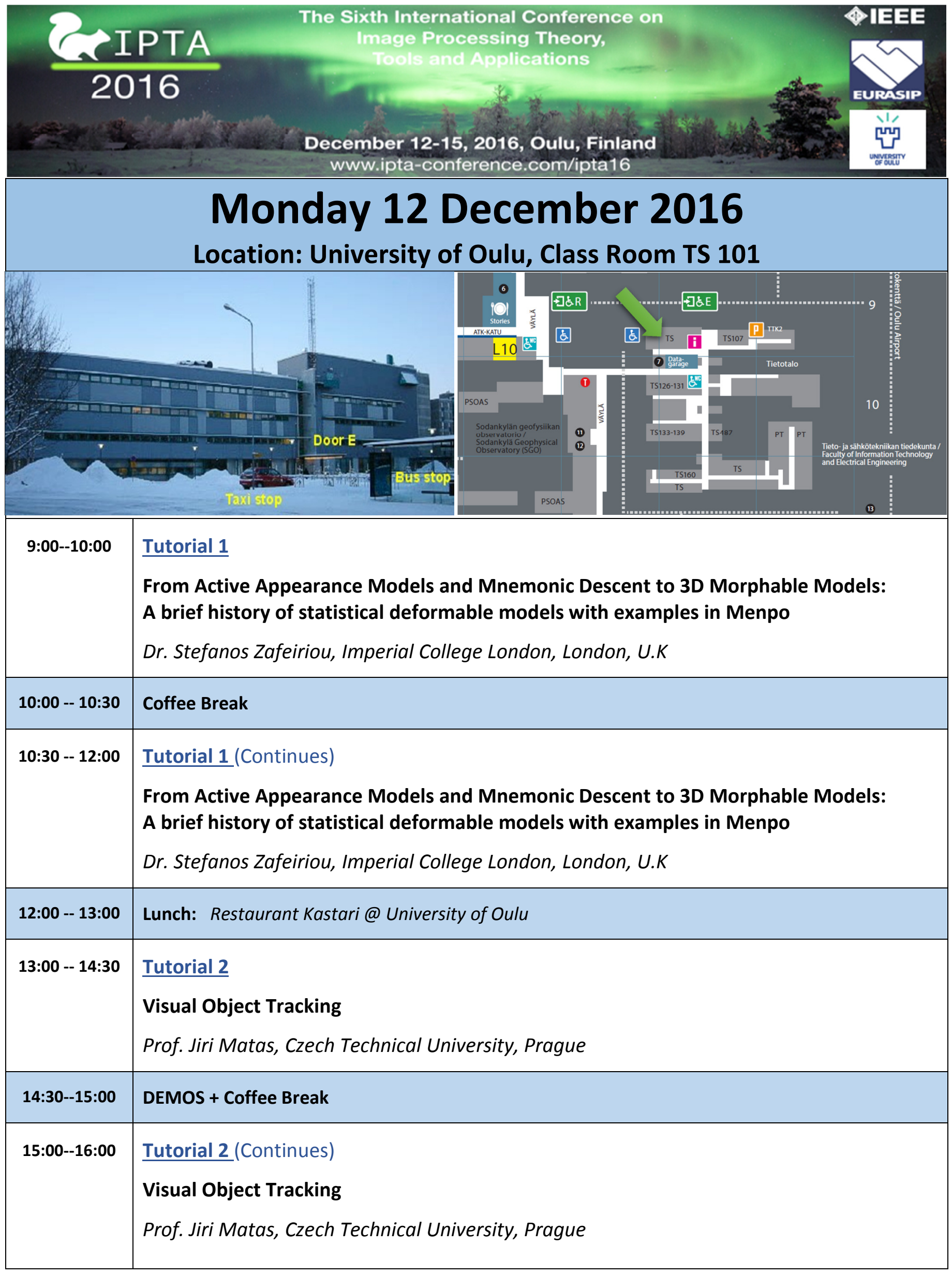




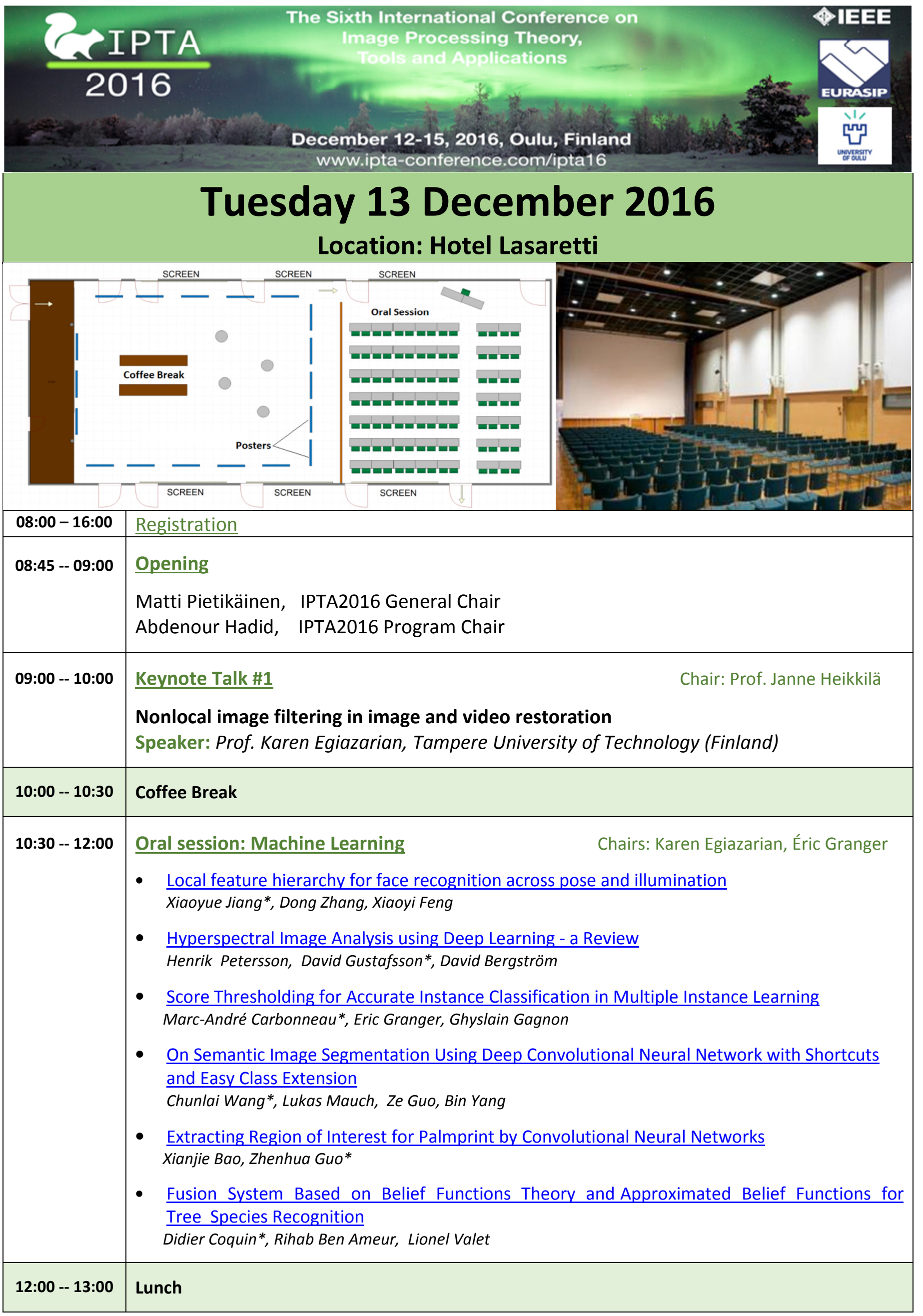




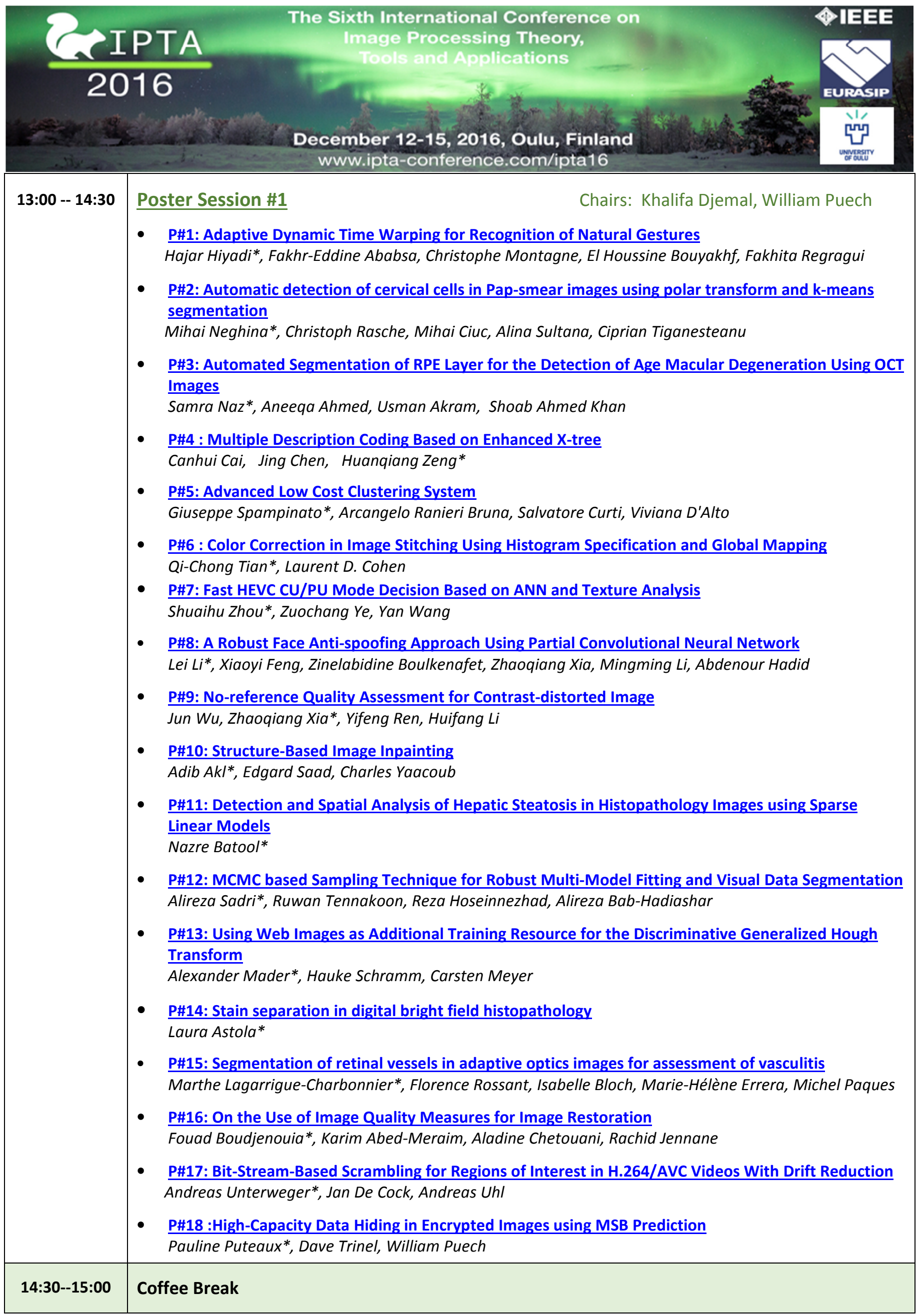




\begin{tabular}{|c|c|}
\hline & $\begin{array}{l}\text { December 12-15, 2016, Oulu, Finland } \\
\text { www.ipta-conference.com/ipta16 }\end{array}$ \\
\hline 15:00--16:30 & $\begin{array}{l}\text { Oral session: Advances in Medical Imaging Chairs: Rachid Jennane, Su Ruan } \\
\text { - } \frac{\text { Diffusion Weighted Imaging of Prostate Cancer: Prediction of Cancer using Texture Features }}{\text { from Parametric Maps of the Monoexponential and Kurtosis functions }} \\
\text { Ileana Montoya Perez*, Jussi Toivonen, Parisa Mohavedi, Harri Merisaari, Marko pesola, Pekka Taimen, } \\
\text { Peter J. Boström, Aida Kiviniemi, Hannu J. Aronen, Tapio Pahikkala, Ivan Jambor } \\
\text { - } \frac{\text { Automatic Modeling and Classification of Vitreomacular Traction Pathology Stages }}{\text { Agnieszka Stankiewicz*, Tomasz Marciniak Adam Dabrowski, Marcin Stopa Piotr Rakowicz, Elzbieta }} \\
\text { Marciniak, } \\
\text { - } \frac{\text { Automatic monitoring system for the detection and evaluation of the evolution of }}{\text { hemangiomas }} \\
\text { - Eatalina Neghina*, Marta Zamfir, Mihai Ciuc, Alina Sultana, Maria Popescu } \\
\text { Evaluation of feature sensitivity to training data inaccuracy in detection of retinal lesions } \\
\text { - Lensu* } \\
\text { - Incorporanen, Antti Hannuksela, Ela Claridge, Pauli Fält, Markku Hauta-Kasari, Hannu Uusitalo, Lasse } \\
\text { Michael Gadermayr*, Hubert Kogler, Maximilian Karla, Andreas Vécsei, Andreas Uhl, Dorit Merhof } \\
\frac{\text { Classification of mammographic microcalcification clusters using a combination of topological }}{\text { and location modelling }} \\
\text { Oluwaseun Ashiru*, Reyer Zwiggelaar }\end{array}$ \\
\hline 19:00 - 21:00 & ence Reception @ Oulu City Hall \\
\hline
\end{tabular}




\begin{tabular}{|c|c|}
\hline & 6 December 12-15, 2016, Oulu, Finland \\
\hline & Location: Hotel Lasaretti \\
\hline 08:30--16:00 & Registration \\
\hline 09:00--10:00 & $\begin{array}{l}\text { Keynote Talk \#2 } \\
\text { Deep Learning Networks for Computer Vision and Pattern Recognition } \\
\text { Speaker: Prof. Rama Chellappa, University of Maryland, College Park }\end{array}$ \\
\hline 10:00--10:30 & Coffee Break \\
\hline 10:30--12:00 & $\begin{array}{l}\text { Oral session: Biometrics and Forensics } \\
\text { - } \frac{\text { Improved Interpolation Kernels for Super resolution Algorithms }}{\text { Pejman Rasti*, Olga Orlova, Gert Tamberg,; Cagri Ozcinar, Kamal Nasrollahi, Thomas Moeslund, }} \\
\text { Gholamreza Anbarjafari } \\
\text { - } \frac{\text { On the use of skin texture features for gender recognition: an experimental evaluation }}{\text { Francesco Bianconi*, Fabrizio Smeraldi, Maryam Abdollahyan, Perry Xiao }} \\
\text { - } \frac{\text { Phylogeny of JPEG images by ancestor estimation using missing markers on image pairs }}{\text { Noé Le Philippe*, William Puech, Christophe Fiorio }} \\
\text { - } \frac{\text { Resource-Efficient Latent Fingerprint Age Estimation for Adhoc Crime Scene Forensics: Quality }}{\text { Assessment of Flat Bed Scans and Statistical Features }} \\
\text { Ronny Merkel*, Jana Dittmann, Claus Vielhauer } \\
\frac{\text { Investigation of Adaptive Local Threshold Segmentation in Context of 3D-Handwriting }}{\text { Forensics }} \\
\text { Michael Kalbitz }{ }^{*} \text {, Tobias Scheidat, Claus Vielhauer } \\
\frac{\text { Face spoofing detection with image quality regression }}{\text { Haoliang Li*, Shiqi Wang, Alex Kot }}\end{array}$ \\
\hline 12:00--13:00 & Lunch \\
\hline 13:00--14:30 & $\begin{array}{l}\text { Poster session \#2 } \\
\text { - } \quad \frac{\text { P\#19: De-Identifying People in Videos using Neural Art }}{\text { Karla Brkic*, Ivan Sikiric, Tomislav Hrkac, Zoran Kalafatic }} \\
\text { - } \frac{\text { P\#20: Efficient BSIF-based Near-Infrared Iris Recognition }}{\text { Christian Rathgeb*, Florian Struck, Christoph Busch }} \\
\text { - } \frac{\text { P\#21: De-Convolutional Auto-Encoder for Enhancement of Fingerprint Samples }}{\text { Patrick Schuch*, Simon Schulz, Christoph Busch, }} \\
\text { - } \frac{\text { P\#22: Multi-Biometric Template Protection - A Security Analysis of Binarized Statistical Features for }}{\text { Bloom Filters on Smartphones }} \\
\text { Martin Stokkenes*, Raghavendra Ramachandra, Morten Sigaard, Kiran Raja, Christoph Busch, Marta } \\
\text { Gómez-Barrero }\end{array}$ \\
\hline
\end{tabular}




\begin{tabular}{|c|c|}
\hline & $\begin{array}{l}\text { December 12-15, 2016, Oulu, Finland } \\
\text { www.ipta-conference.com/ipta16 }\end{array}$ \\
\hline & 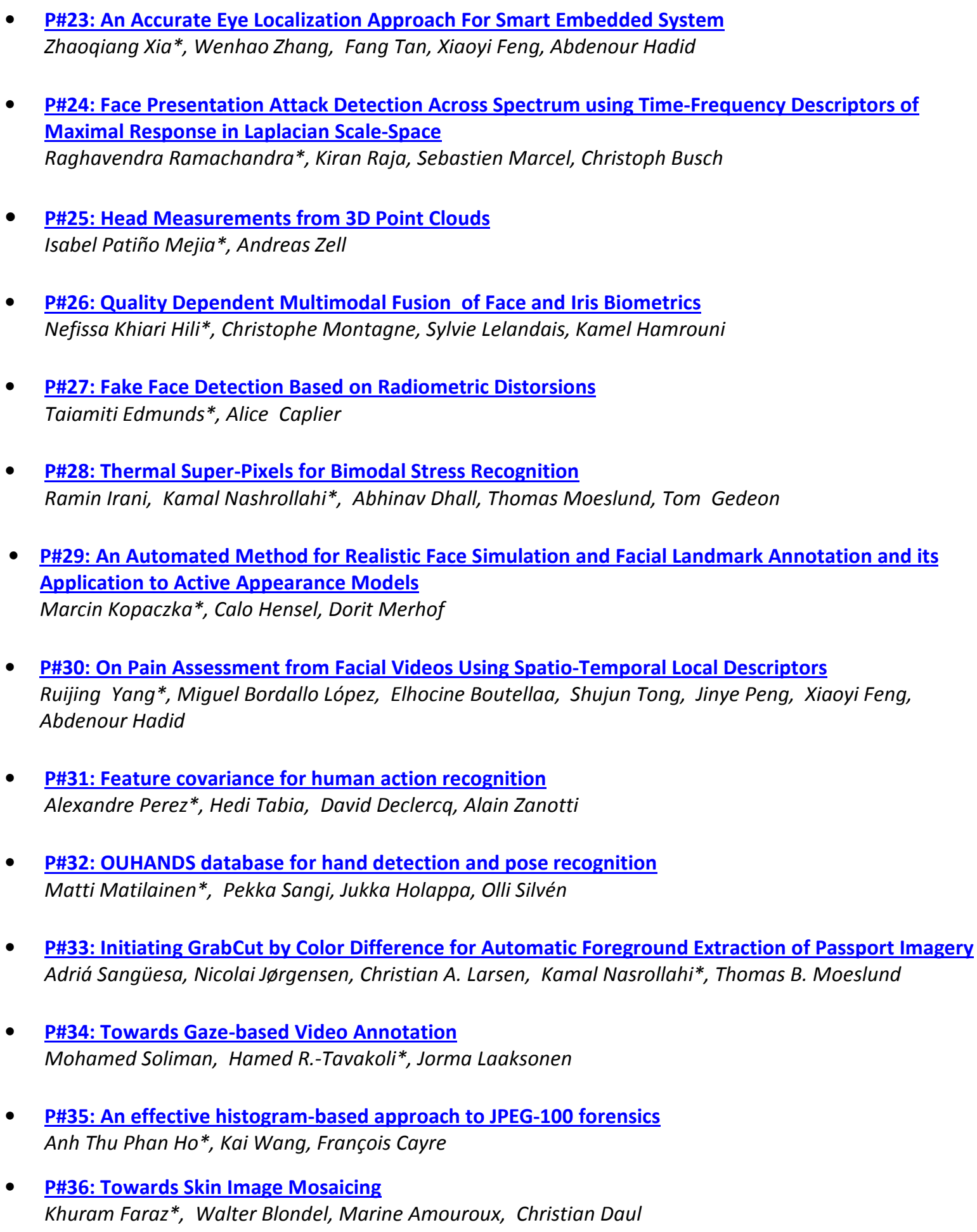 \\
\hline 14:30--15:00 & Coffee Break \\
\hline
\end{tabular}




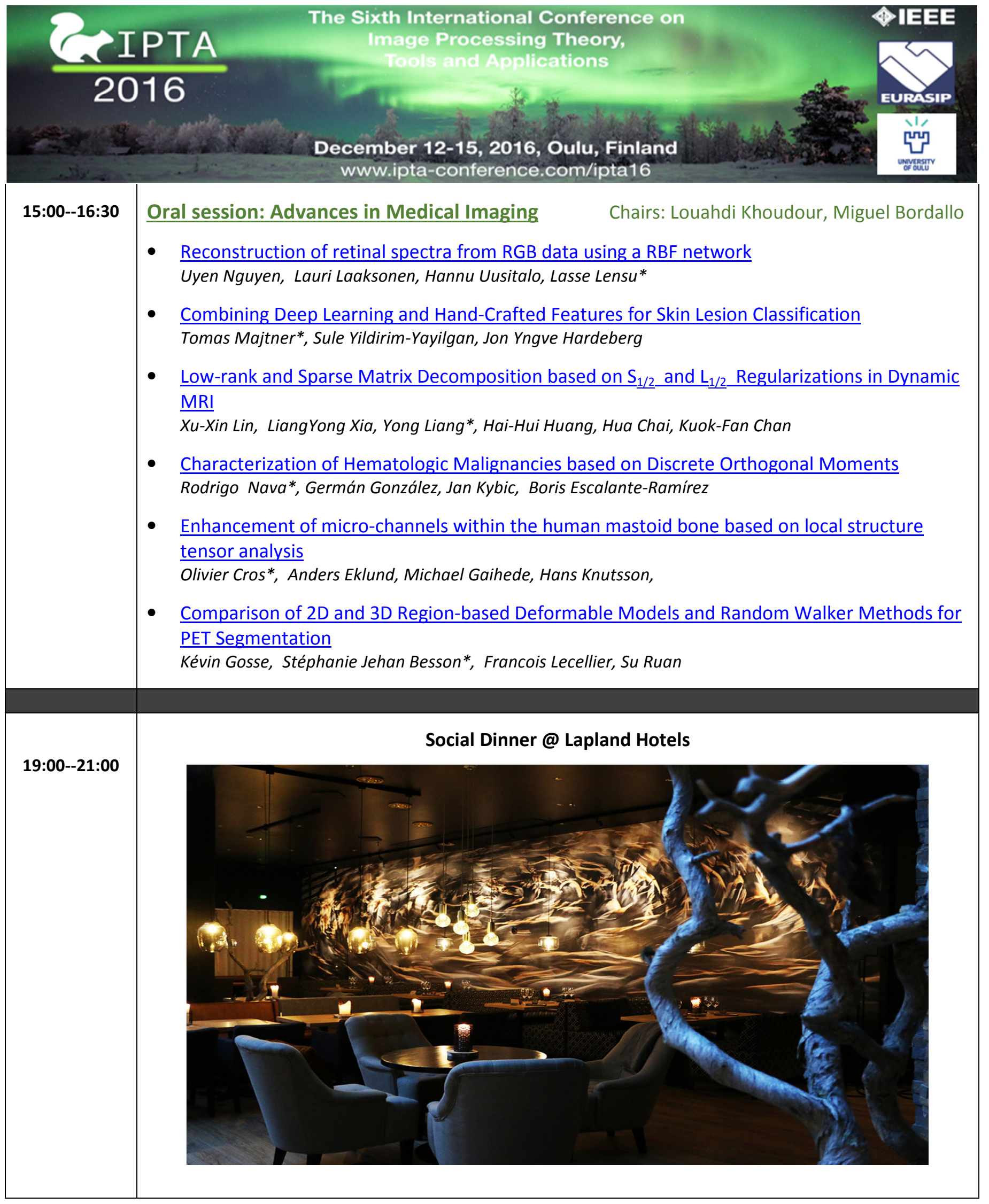




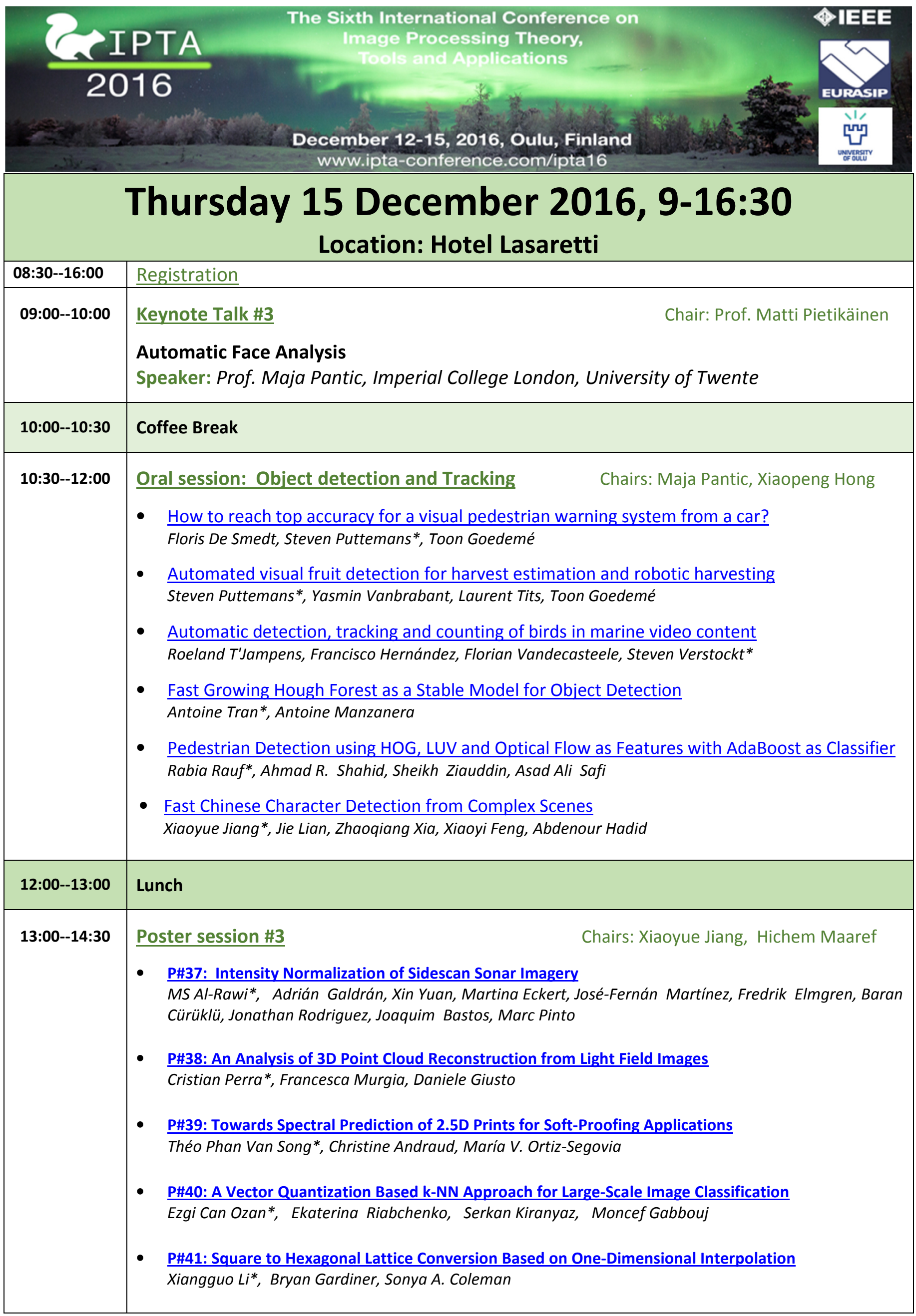




\begin{tabular}{|c|c|}
\hline & $\begin{array}{c}\text { December 12-15, 2016, Oulu, Finland } \\
\text { www.ipta-conference.com/ipta16 }\end{array}$ \\
\hline & 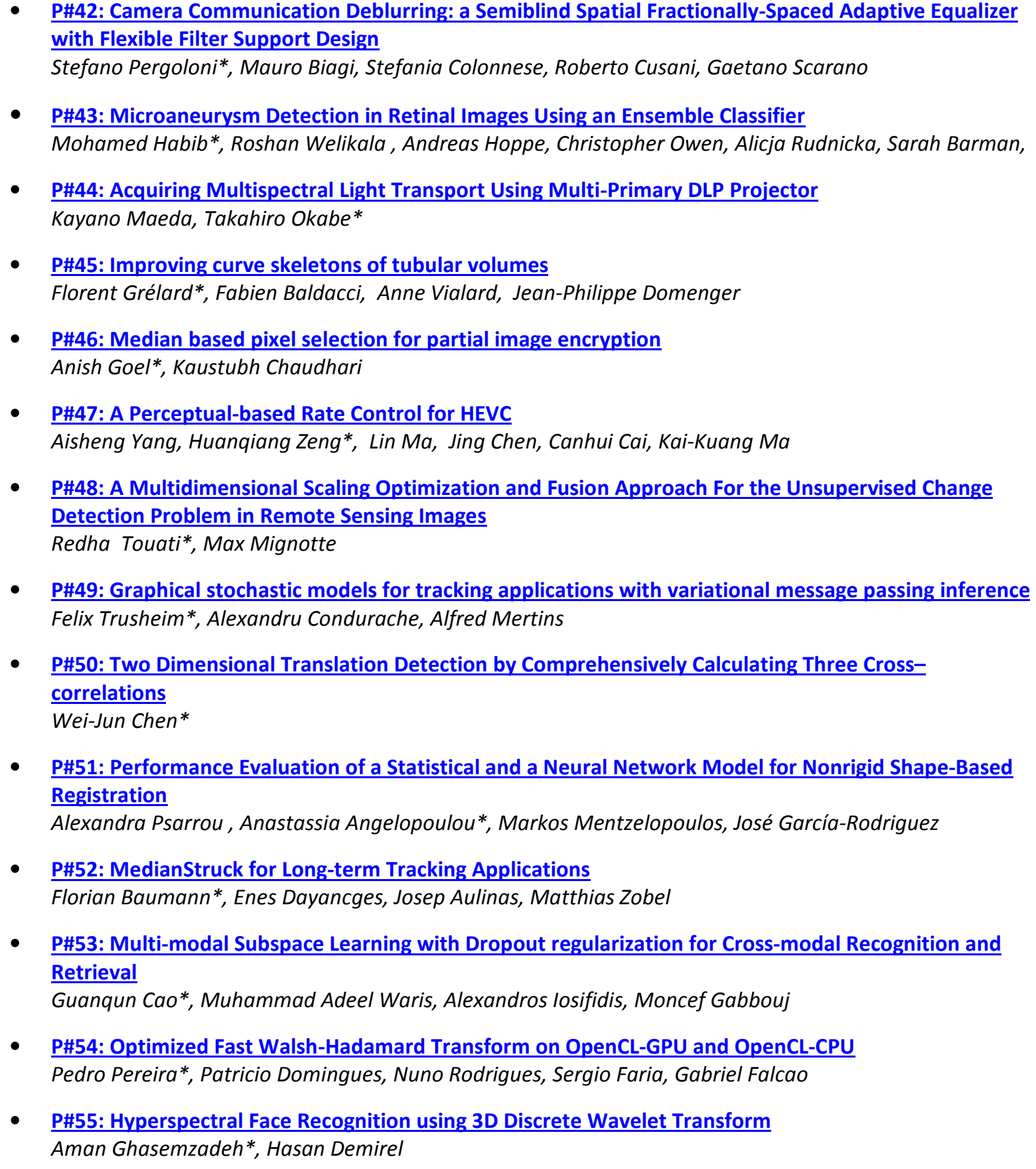 \\
\hline 14:30--15:00 & Coffee Break \\
\hline
\end{tabular}




\begin{tabular}{|c|c|}
\hline & $\begin{array}{l}\text { The Sixth International Conference on } \\
\text { Image Processing Theory, } \\
6 \text { Tools and Apolications } \\
\text { December 12-15, 2016, Oulu, Finland } \\
\text { www.ipta-conference.com/ipta16 }\end{array}$ \\
\hline 15:00--16:30 & $\begin{array}{l}\text { Oral Session : Best Reviewed Papers } \\
\text { - } \text { Unsupervised Deep Hashing for Large-scale Visual Search } \\
\text { Zhaogiang Xia*, Xiaoyi Feng, Jinye Peng, Abdenour Hadid } \\
\text { - } \frac{\text { Single image super-resolution reconstruction in presence of mixed Poisson-Gaussian noise }}{\text { Buda Bajic*, Joakim Lindblad, Natasa Sladoje }} \\
\text { - } \frac{\text { Fast feature matching for detailed point cloud generation }}{\text { Daniel Berjón*, Rafael Pagés, Francisco Morán }} \\
\text { - } \frac{\text { CNN Transfer Learning for the Automated Diagnosis of Celiac Disease }}{\text { Georg Wimmer*, Andreas Vécsei, Andreas Uhl }} \\
\text { - } \frac{\text { Prototype-based Class-Specific Nonlinear Subspace Learning for Large-Scale Face Verification }}{\text { Alexandros losifidis*, Moncef Gabbouj }} \\
\text { - } \frac{\text { RealSense = Real Heart Rate: Illumination Invariant Heart Rate Estimation from Videos }}{\text { Jie Chen*, Zhuoqing Chang, Qiang Qiu, Xiaobai Li, Guillermo Sapiro, Alex Bronstein, Matti Pietikäinen }}\end{array}$ \\
\hline 16:30--17:00 & Chair: Matti Pietikäinen \\
\hline
\end{tabular}

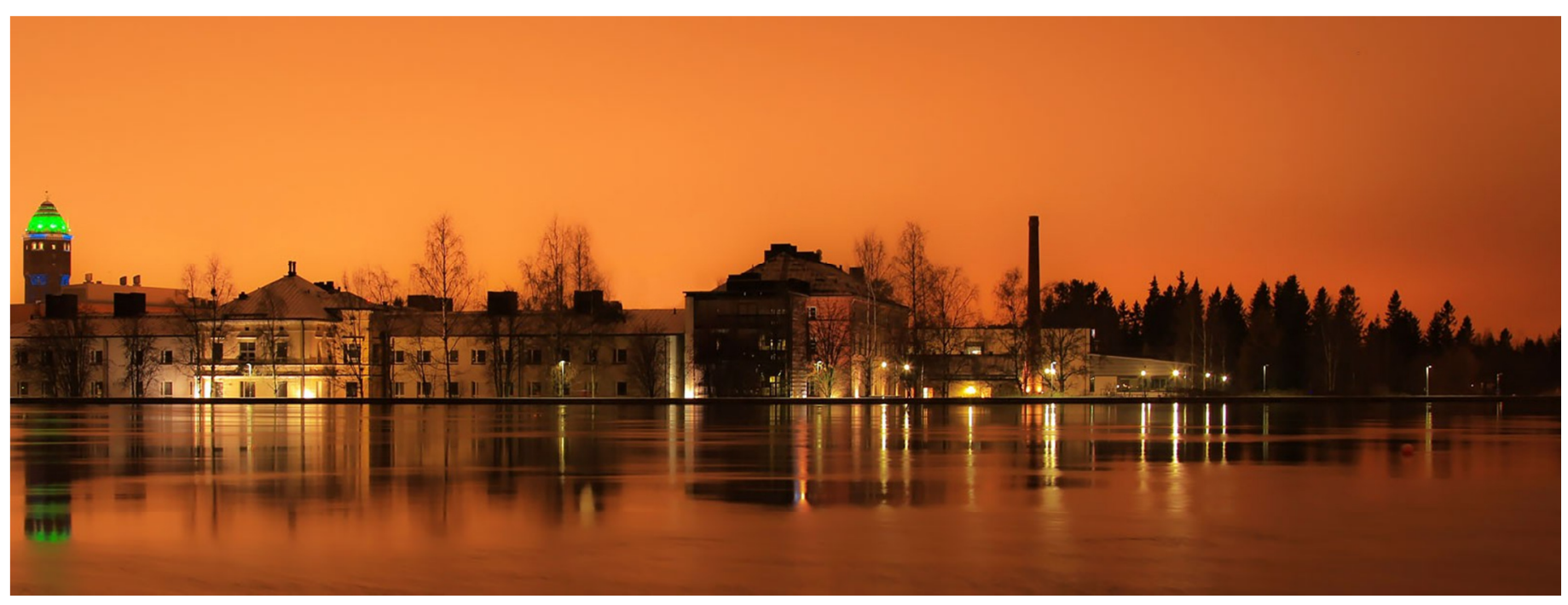




\section{Notes}

- The tutorials on Monday 12 December will be held at the classroom TS101 at University of Oulu (NOT at the conference Venue!!!). Please see directions at the IPTA2016 website.

- The main conference will be held at Hotel / Conference Centre Lasaretti (downtown!!)

- Oral presentation: 15 minutes (10-12 minutes for presentation and 3-5 minutes for questionsanswers). The session chairs are advised to stop the presenters after 15 minutes!!

- Posters: The poster stand size will be around $1470 \mathrm{~mm} \times 1470 \mathrm{~mm}$. Format A0 (Vertical, portrait) will fit fine. A0 paper size $=841 \mathrm{~mm}$ by $1189 \mathrm{~mm}$ (or $33.1 \mathrm{in} \times 46.8 \mathrm{in}$ ) $(:)$

- The dinner will be held at Lapland Hotels (at a walking distance from the conference venue).

- There will be a best reviewed paper session on Thursday 15 December 15:00 -- 16:30

- The best paper awards will be announced on Thursday 15 December at 16:30

\section{Program at glance}

\begin{tabular}{|c|c|c|c|c|}
\hline & $\begin{array}{c}\text { Monday 12 Dec } \\
\text { (University of Oulu - TS101) }\end{array}$ & $\frac{\text { Tuesday } 13 \text { Dec }}{\text { (Hotel Lasaretti) }}$ & $\frac{\text { Wednesday } 14 \text { Dec }}{\text { (Hotel Lasaretti) }}$ & $\begin{array}{l}\text { Thursday } 15 \text { Dec } \\
\text { (Hotel Lasaretti) }\end{array}$ \\
\hline 08:30- & & Registration Open & Registration Open & Registration Open \\
\hline 09:00-10:00 & Tutorial 1 & Keynote Talk 1 & Keynote Talk 2 & Keynote Talk 3 \\
\hline $10: 00-10.30$ & Coffee Break & Coffee Break & Coffee Break & Coffee Break \\
\hline 10:30 - 12:00 & Tutorial 1 & Oral Session & Oral Session & Oral Session \\
\hline 12:00-13:00 & Lunch & Lunch & Lunch & Lunch \\
\hline 13:00-14:30 & Tutorial 2 & Poster Session & Poster Session & Poster Session \\
\hline 14:30 - 15:00 & Demos + Coffee Break & Coffee Break & Coffee Break & Coffee Break \\
\hline $15: 00-16: 30$ & Tutorial 2 & Oral Session & Oral Session & Oral Session \\
\hline 19:00-21:00 & & Reception & Dinner & \\
\hline
\end{tabular}

